\title{
Fellowship for the Interpretation of Genomes
}

National Cancer Institute

\section{Source}

National Cancer Institute. Fellowship for the Interpretation of Genomes. NCI Thesaurus.

Code C48833.

A non-profit organization specializing in bioinformatics tool development and comparative genomics research. 